\title{
Sustitución y uso de medicamento de humana para el tratamiento de leishmania en perros
}

\author{
Sandra María López Fernández \\ Veterinaria. Investigadora del CIMES (Centro de Investigaciones Médico Sanitarias. Facultad de Medicina, Universidad de Málaga).
}

\section{PALABRAS CLAVE}

Leishmania, salud pública, terapia, refugios, formulación, receta veterinaria, miltefosina, antimoniato de meglumina, medicina veterinaria

\section{ABREVIATURAS}

OMS: Organización Mundial de la Salud

\section{KEYWORDS}

Leishmania, public health, drug therapy, shelters, formulation, veterinary prescriptions, miltefosine, meglumine antimoniate, veterinary medicine

\section{RESUMEN}

Antecedentes: la leishmaniosis es una zoonosis vectorial que en humanos inmunodeprimidos presenta forma sistémica, estando la forma cutánea infradiagnosticada.

El tratamiento para el portador tiene un elevado coste. Propietarios y centros de adopciones carecen de recursos, pero no tratar o prevenir implica tener a un portador de una zoonosis disponible para el vector.

Por ello existe una tendencia a la sustitución por la presentación de humana por los agentes implicados, adelantándose a los responsables en materia de salud pública.

Objetivos: conocer la incidencia de animales portadores, la proporción de medicamentos prescritos por los veterinarios, las tendencias de búsqueda en internet de medicamentos para prevención y el tratamiento.

Metodología: análisis serológico por técnica de inmunoensayo de 255 perros, aplicación de precios de mercado a los resultados, encuesta sobre la prescripción de veterinarios clínicos y análisis de tendencias de búsqueda de información en internet entre usuarios.

Resultados: 38 perros resultaron ser portadores inaparentes (infectados, pero no enfermos) y 28 portadores enfermos. El resto son no portadores. Se obtuvo una diferencia de precio de más del $50 \%$ en el tratamiento entre medicamentos de humana o genéricos y el de veterinaria. En la prevención la diferencia es 11 veces el precio veterinario frente al de humana.

Los veterinarios prescriben correctamente, pero informan de otras opciones más baratas y la tendencia de búsqueda en internet está orientada al precio y a la sustitución.

Conclusión: el precio es la principal causa de sustitución del medicamento prescrito y la legislación no está atendiendo la relevancia de esta zoonosis. Las autoridades sanitarias han de valorar las necesidades reales en materia de salud pública.

\section{Substitution and use of human drugs for the treatment of canine lesihamniasis}

\section{ABSTRACT}

Background: Leishmaniasis is a vectorial zoonosis with systemic presentation in immunosuppressed humans, the cutaneous form is underdiagnosed.

A lot of owners and Rescue kennels can not pay the treatment because it is very expensive, but not treating or not preventing is dangerous for Public Health.

Therefore, there is a tendency to replace the veterinary drug by the human drug form, ahead of those responsible for Public Health.

Objectives: To know the disease carrier animals, the prescription tendencies of the veterinarians, the tendencies of search in Internet of drugs for prevention and treatment.

Methods: Blood test of 255 animals, application of market prices to the results, survey on the prescription of clinical veterinarians and analysis of Internet search trends among users.

Results: 38 dogs were founded inapparent carriers (infected, but not ill) and 28 sick carriers. The rest were non-carriers. A price difference of more than $50 \%$ was obtained in the treatment between human or generic drugs and that of veterinary medicine. About the prevention, the difference is 11 times the veterinary price compared to that of humans.

Veterinarians prescribe correctly but report other cheaper options and the search trend on the internet is price and replacement oriented.

Conclusion: The price is the main cause of substitution and the legislation is not agree the relevance of this zoonosis. The health authorities must assess the real needs in terms of Public Health. 


\section{Antecedentes}

La leishmaniosis es una zoonosis vectorial endémica en nuestro país, abarcando cada vez más territorio afectado. Está provocada por Leishmania infantum, protozoo kinetoplástido parásito intracelular obligado. Está considerada como zoonosis emergente por la Organización Mundial de la Salud (OMS) (1) y constituye un grave problema de salud en perros domésticos. Se transmite por la picadura de la hembra de Phlebotomus Spp.

En seres humanos del primer mundo sanos, la picadura puede provocar una reacción local leve que suele pasar desapercibida al paciente. Tiene tres formas de presentación: la cutánea, la mucocutánea y la visceral (la más grave). Sólo en casos en que el paciente tiene comprometido su sistema inmunológico (sida, trasplantados, terapias inmunosupresoras, ancianos, neonatos), el parásito causa enfermedad (2). Estos individuos han de ser tenidos en cuenta a la hora de valorar las necesidades preventivas y terapéuticas.
La prevalencia de la exposición al parásito es muy variable sobre todo dependiendo de la situación geográfica y los microclimas locales. Puede oscilar entre el $10 \%$ (zonas ventosas y frías) y el $80 \%$ (zonas más cálidas o protegidas) y según los animales que están o han estado expuestos en protectoras que solo distan pocos kilómetros unas de otras. Más de la mitad de ellos desarrollará la enfermedad en algún momento de su vida (2). Esto se debe a que el parásito, al tener un vector volador como transmisor, es muy dependiente del medio, tanto para el desplazamiento del mosquito como para su mantenimiento y cría en el ambiente. A lo largo de este estudio se han encontrado municipios con una prevalencia altísima y, a pocos kilómetros, otros municipios con una prevalencia muy baja, siendo la diferencia la geografía y altura del municipio.

El tratamiento prescrito por los veterinarios en España varía de un profesional a otro, pero básicamente consta de un leishmaniostático (alo- purinol), uno o dos inmunomoduladores (como la domperidona) y un leishmanicida, ya sea un antimonial pentavalente (comercializado bajo dos formas, Glucantime ${ }^{\circledR}$ y Antishmania ${ }^{\circledR}$ ) o a base de miltefosina (comercializado como Milteforán $\left.{ }^{\circledR}\right)$ (3)(4).

Ambos leishmanicidas tienen un alto coste al comprarlos en la farmacia comunitaria y es muy común que los propietarios consulten en internet opciones más baratas y luego pidan a los profesionales si pueden prescribir el de uso humano por ser más barato (5).

La Ley del medicamento veterinario especifica que la prescripción de medicamentos debe seguir un orden de prioridades (Real Decreto 1132/2010, de 10 de septiembre (10), por el que se modifica el Real Decreto 109/1995, de 27 de enero, sobre medicamentos veterinarios). Según la cascada de prescripción (figura A), la opción más cara sería la primera y la obligatoriamente usada para el tratamiento y la prevención de la leishmaniosis canina.

\section{RD1132/201010. Artículo 81. Prescripciones excepcionales por vacío terapéutico en animales no productores de alimentos.}

Cuando no existan medicamentos veterinarios autorizados para una enfermedad, en una especie animal no productora de alimentos, incluidas las domésticas, el veterinario podrá, de forma excepcional y bajo su responsabilidad personal directa, en particular para evitar sufrimientos inaceptables, tratar al animal o animales afectados con:

$1^{\text {er }}$ nivel

a. Un medicamento veterinario, con similar efecto terapéutico al deseado, autorizado en España para esa misma especie animal pero para otra enfermedad, ó

b. Un medicamento veterinario, con similar efecto terapéutico al deseado, autorizado en España para esa misma enfermedad pero en otra especie animal.

$2^{\circ}$ nivel

a. Un medicamento de uso humano autorizado en España, ó

b. Un medicamento veterinario, con similar efecto terapéutico al deseado, autorizado en otro Estado de la Unión Europea, para esa especie animal o para otras, y para esa enfermedad u otras. En este caso, el veterinario comunicará con antelación suficiente su intención de adquirir el medicamento veterinario a los servicios competentes de su comunidad autónoma, la cual podrá prohibir su uso por razones de sanidad animal o salud pública mediante resolución notificada a dicho veterinario en el plazo de 5 dias. Para mayor información sobre el procedimiento de adquisición de estos medicamentos consultar la Nota Informativa sobre Prescripción Excepcional elaborada por la Agencia Española de Medicamentos y Productos Sanitarios y por el Ministerio de Agricultura, Alimentación y Medio Ambiente, disponible en las páginas Web de ambos organismos.

$3^{\text {er }}$ nivel
a. Una Fórmula Magistral, ó
b. Un Preparado Oficinal, ó
c. Una Autovacuna.

Figura A Resumen de la Ley del medicamento veterinario (Real Decreto 1132/2010), de la llamada "prescripción en cascada". https://www.aemps.gob.es/medicamentosVeterinarios/saludVeterinaria/prescripcion_cascada_faq.htm 
Los refugios y centros de adopciones son focos de la enfermedad para otros perros y para los humanos susceptibles, pero carecen, en su mayoría, de los medios para prevenirla y mucho menos tratarla. Igual ocurre con los propietarios de animales con bajos ingresos y sus animales, si son infectados, representan un riesgo sanitario para la población susceptible. El sacrifico de los animales enfermos no es viable, ya que estudios realizados en Brasil demuestran que no es eficaz (6).

El problema que plantea el tratamiento de esta enfermedad es que, si los propietarios no pueden hacerse cargo del tratamiento, tenemos a un animal portador de una zoonosis que es contagioso si es picado por un Phlebotomo (7).

Muchos propietarios, con o sin ayuda veterinaria, al igual que refugios y centros de adopción, utilizan medicamentos para uso humano en el tratamiento y prevención (8) de leishmaniosis por su bajo precio comparado con el mismo principio activo comercializado para uso veterinario, siendo el clínico el único que se encuentra con las manos atadas a la hora de enfrentar la enfermedad desde el punto de vista epidemiológico.

Se hace necesario establecer unas prioridades a la hora de proporcionar tratamiento a estos animales y que las autoridades sanitarias se hagan cargo del riesgo epidemiológico y se adapten a las necesidades en materia de prevención del contagio a las personas.

Farmacéuticos, veterinarios y legisladores han de ponerse de acuerdo y ponderar cuáles deben ser las medidas más idóneas para proteger a las personas susceptibles.

\section{Objetivos}

- Conocer la cantidad de animales portadores.

- Saber los medicamentos que prescriben los veterinarios clínicos.

- Conocer las tendencias de búsqueda de medicamentos que los usuarios hacen en internet para prevención y tratamiento de leishmaniosis.

- Elaborar una tabla con los casos negativos, positivos y enfermos en varios refugios y aplicar los gastos tanto de tratamiento como de prevención en estos centros, com- parando los costes económicos $\mathrm{y}$ usando medicación de veterinaria y de humanos.

- Mostrar a los farmacéuticos, veterinarios y legisladores la importancia de adecuar las leyes y normas a la situación y necesidades reales de esta zoonosis.

\section{Metodología}

Identificación de animales portadores y cálculo de costes:

Extracción, a lo largo de 3 años, de muestras sanguíneas en 255 perros procedentes de varios refugios de animales y zoosanitarios municipales de la provincia de Málaga. La extracción se realiza de vena cefálica y la sangre es posteriormente centrifugada y el suero separado para realizar una determinación de anticuerpos IgG anti-Leishmania infantum analizado por Inmunofluosflorescencia Indirecta (IFI). Tanto la sensibilidad como la especificidad de la prueba IFI son muy altas, cercanas al 100 y por ello está considerada por la Organización Internacional de Epizootias como la prueba diagnóstica de referencia (9).

Los resultados se expresan en fracciones de dilución. Entre 1/40 y $1 / 80$ se considera animal expuesto y a partir de 1/160 se considera enfermo. Por debajo de 1/40 se considera sano.

Se separan pues a los sujetos en tres grupos: expuestos (portadores inaparentes), enfermos (portadores enfermos) y sanos (no portadores) y se calculan los costes de prevención para el primero y el tercero, así como del tratamiento para el segundo, usando medicamentos de prescripción veterinaria obtenidos a partir de un distribuidor de medicamentos veterinarios y medicamentos de prescripción humana a partir del precio disponible en la web de consulta www.vademecum.es. Luego se comparan los costes de la compra de los medicamentos tanto de uso humano como de uso veterinario entre ambos grupos.

Se calculó un peso medio de $20 \mathrm{~kg}$ por cada animal y se multiplicó por el precio del tratamiento.

El precio de los medicamentos para ese grupo de animales fue calculado por animal y por el grupo completo por cada medicamento.
Encuesta sobre la prescripción de veterinarios clínicos

En dos grupos de estudio, tendencias de prescripción de principios activos y preferencias a la hora de prescribir medicamentos entre uso humano y uso veterinario.

Se elabora una lista de preguntas con respuestas cerradas y se muestran a los veterinarios.

\section{Encuesta A}

Grupo de 225 profesionales veterinarios especializados en clínica de pequeños animales, con las siguientes opciones de respuesta a la pregunta:

\section{“¿Qué leishmanicida usa en animales} enfermos?"

a) Siempre uso antimonial.

b) He dejado de usar miltefosina por las recidivas.

c) La miltefosina me da recidivas antes del primer año.

d) Siempre uso miltefosina.

e) Antimonial en títulos altos, miltefosina en títulos bajos.

\section{Encuesta B}

Grupo de 123 profesionales con las siguientes opciones a la pregunta (102 veterinarios no contestaron a la segunda pregunta):

“¿Prescribe usted medicamentos de humana para el tratamiento o la prevención de leishmaniosis canina?"

a) Nunca los prescribo.

b) No los prescribo pero informo de su existencia en casos muy excepcionales.

c) No los prescribo pero siempre informo de esa opción.

d) Sólo en casos muy excepcionales (bajos ingresos, protectoras y refugios, casos extremos).

e) Sí los prescribo.

\section{Análisis de tendencias de} búsqueda en internet

Entre los usuarios que pueden dar una idea de lo que los propietarios buscan, realizado mediante la aplicación Google Trends (Google Insight for Search), así como las tendencias de búsqueda por términos concretos, relacionados con tratamientos y precios en leishmaniosis. Los términos analizados son miltefosina, Glucantime $^{\circledR}$, Leishguard ${ }^{\circledR}$, Milteforán ${ }^{\circledR}$. Búsqueda realizada en el mes de noviembre de 2018. 


\section{Resultados}

Identificación de animales portadores y cálculo de costes

Se analizaron muestras procedentes de 255 animales clasificándose a los perros de la siguiente manera (figura B):

- Perros sanos: aquellos con un título de anticuerpos inferior a 1-40.

- Portadores: perros que presentaron titulaciones entre 1-40 y 1-160.

- Enfermos: sujetos que mostraron títulos por encima de 1-160.

Respecto a los 189 sujetos sanos no portadores, si un refugio quisiera dar un tratamiento preventivo a todos estos animales para evitar el contagio debería administrar domperidona $0,5 \mathrm{mg} / \mathrm{kg} /$ 24 horas durante 28 días con el coste de 18,70 euros por animal si se tratase con Leishguard ${ }^{\circledR}$ frente a los 2.13 euros por animal en el caso de la domperidona genérico (gráfica 1).

Los 38 perros expuestos deben ser tratados de forma preventiva con el mismo producto y la misma dosificación que los sanos, existiendo una diferencia de 629,66€ entre los $80,94 €$ que costaría si se usa la formulación de sustitución con respecto a los $710,60 €$ si se usa la formulación veterinaria (tabla 1).

Por último, los 28 animales enfermos, deberían recibir un tratamiento combinado a base de un leishmanicida (o antimonial pentavalente o miltefosina) junto a domperidona y alopurinol. Como el alopurinol no existe aún en formulación veterinaria, no se ha incluido en este estudio al no representar diferencia en la comparación de costes, es una constante (tabla 2).

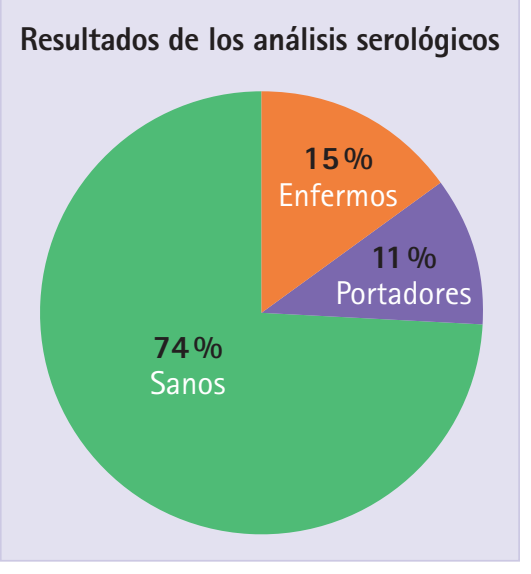

Figura B Distribución de perros sanos respecto a los portadores (portadores inaparentes y portadores enfermos) en la población estudiada.

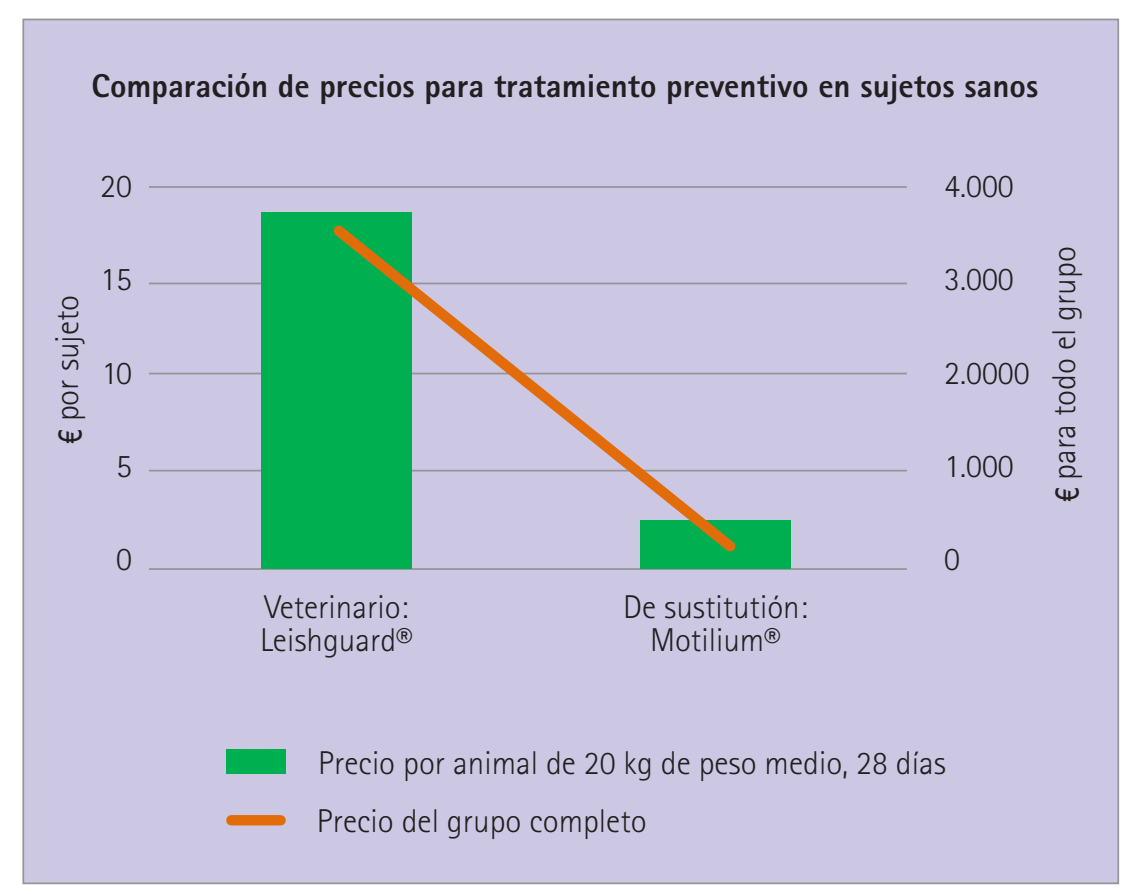

Gráfica 1. Costes de tratamiento preventivo en sujetos sanos

La domperidona de uso veterinario representa para un refugio un coste tanto individual como colectivo de más del $100 \%$ si usan la presentación de uso veterinario. La gráfica muestra la diferencia en los costes a asumir en estos colectivos, desde los casi 4.000 euros de un colectivo tratado con Leishguard ${ }^{\circledR}$ respecto al los menos de 1.000 si se usa Motilium ${ }^{\circledR}$.

Tabla 1 Cálculo de precios para los sujetos portadores no enfermos

\begin{tabular}{|l|l|c|c|}
\hline Principio activo & & $\begin{array}{c}€ / 20 \mathrm{~kg} / \\
\text { Tratamiento completo }\end{array}$ & $\begin{array}{c}38 \text { sujetos } \\
\text { expuestos }\end{array}$ \\
\hline Domperidona & Leishguard $^{\circledR}$ & $18,7 €$ & $710,6 €$ \\
\hline & Motilium $^{\circledR}$ & $2,13 €$ & $80,94 €$ \\
\hline
\end{tabular}

Tabla 2 Cálculo de precios para los sujetos enfermos

Para un animal aislado ya de por sí representa una gran diferencia de precio, pero si comparamos los precios para los grupos completos, una protectora o refugio de animales tendría el doble de gastos comprando el producto de uso veterinario en vez del medicamento de uso humano. En el caso de los preventivos, el coste de la presentación para uso humano es el $10 \%$ del precio total respecto al de uso veterinario.

\begin{tabular}{|c|c|c|c|}
\hline Principio activo & Presentación & $\begin{array}{c}€ / 20 \mathrm{~kg} / \\
\text { Tratamiento completo }\end{array}$ & $\begin{array}{l}28 \text { animales } \\
\text { enfermos }\end{array}$ \\
\hline \multirow[t]{2}{*}{ Miltefosina } & Milteforán ${ }^{\circledR}$ & $168,00 €$ & $4.704,00 €$ \\
\hline & Genérica & $74,70 €$ & $2.091,60 €$ \\
\hline \multirow[t]{2}{*}{ Antimonial } & Glucantime ${ }^{\circledR}$ & $248,00 €$ & $6.944,00 €$ \\
\hline & Antishmania ${ }^{\circledR}$ & $130,60 €$ & $3.656,80 €$ \\
\hline \multirow[t]{2}{*}{ Domperidona } & Leishguard ${ }^{\circledR}$ & $18,70 €$ & $523,60 €$ \\
\hline & Motilium ${ }^{\circledR}$ & $2,13 €$ & $59,64 €$ \\
\hline
\end{tabular}


Encuesta sobre la prescripción de veterinarios clínicos

\section{Encuesta A}

La terapéutica leishmanicida muestra una clara preferencia por el antimonial pentavalente, quedando la miltefosina muy por detrás tras varios años de uso en los que los terapeutas han indicado un gran número de recidivas (gráfica 2).

\section{Encuesta B}

Sustitución de medicamentos veterinarios por presentaciones de humana. Los veterinarios clínicos se resisten a prescribir medicamentos de sustitución y cumplen la ley, pero informan al propietario, ya sea de forma general $(14,6 \%)$ o en caso muy especiales $(22,8 \%)$ lo que suma un $37,4 \%$ de profesionales que están informando de una forma u otra de la existencia de otra presentación mas barata. Una pequeña parte de estos profesionales los está prescribiendo, mientras que los profesionales que ni los prescriben ni informan son menos del $20 \%$ (gráfica 3 ).

\section{Tendencias de búsqueda en} internet

Con respecto al milteforán, a lo largo de los últimos 5 años en las búsquedas en Google ha habido un incremento constante del interés.

En "Temas Relacionados", la comparación con meglumina antimoniato (fórmula química de Glucantime ${ }^{\circledR}$ y Antishmania ${ }^{\circledR}$ ) ha crecido un $120 \%$ en los últimos 5 años y las consultas sobre el precio subieron un $100 \%$. Lo que mas resalta es el incremento de los términos "Glucantime Humano" y "alopurinol" como terapias alternativas (figura C).

En las búsquedas que Google ofrece como relacionadas con "Glucantime" y "leishmaniasis tratamiento farmacológico" aparecen frecuentemente los términos "precio" y "genérico".

\section{Discusión}

Hay preocupación por parte de los propietarios de perros en prevenir el contagio y tratar a los animales infectados, pero los medicamentos veterinarios son caros, lo que lleva a muchos a buscar alternativas más baratas sustituyendo por medicamentos de uso humano con el mismo principio activo. Internet, las redes sociales, incluso los

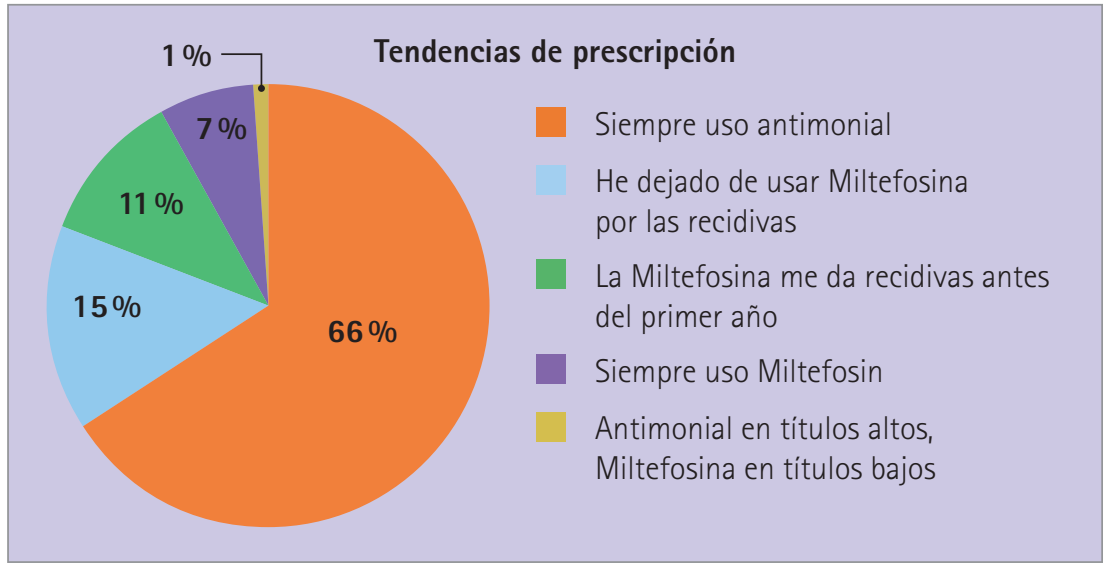

Gráfica 2 Resultado de la encuesta de prescripción ( $N=225)$

"¿Qué medicamento leishmanicida usa en animales enfermos?"

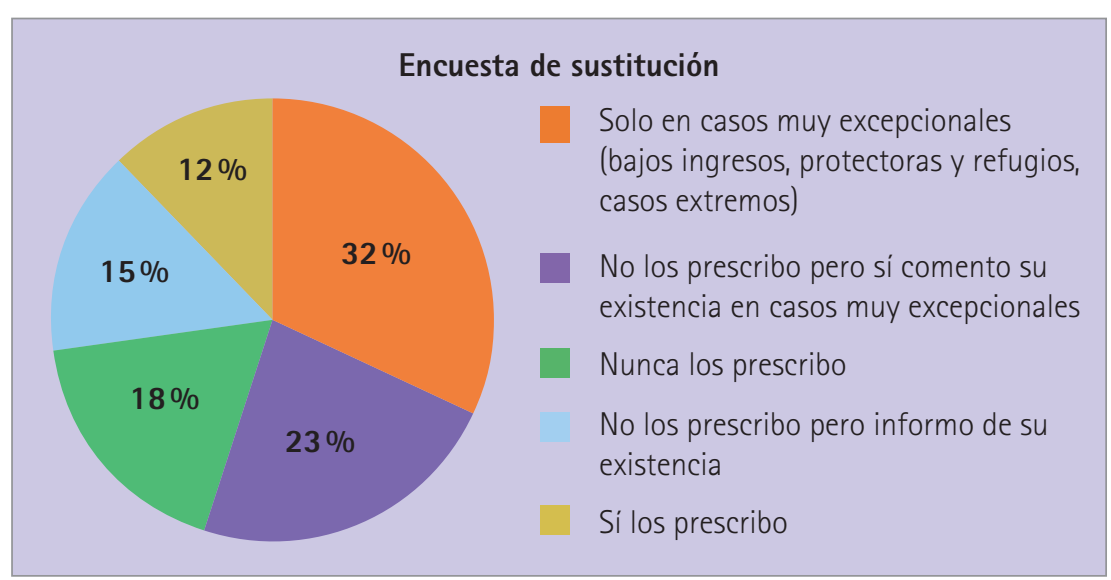

Grafica 3 Resultados de la encuesta de sustitución ( $N=123)$

"¿Prescribe usted medicamentos de uso humano para el tratamiento o la prevención de leishmaniosis canina?"

\begin{tabular}{|l|c|}
\hline - Antimoniato de meglumina & España, Últimos 5 años \\
\hline Consultas relacionadas (?) & En aumento $\quad\langle><$ \\
\hline 1 glucagon & Aumento puntual \\
\hline 2 alopurinol & $+200 \%$ \\
\hline 3 glucantime humano & $+90 \%$ \\
\hline
\end{tabular}

Figura C Las consultas sobre alopurinol han crecido un $200 \%$ en los últimos 5 años y las de Glucantime de uso humano (sustitución del medicamento de uso veterinario) se han incrementado un $90 \%$.

propios veterinarios y farmacéuticos están colaborando en esta búsqueda.

La legislación obliga a dispensar el de veterinaria como primera opción, pero no suministrar el medicamento humano puede hacer que cierto número de animales no sean tratados y queden como portadores que pongan en riesgo a otros reservorios o a los humanos susceptibles.
Muchos centros de acogida usan fórmulas magistrales compradas en formato casi industrial para paliar el elevado coste de los productos. No hacerlo llevaría a muchos animales al contagio y la muerte, pero, además, resultarian ser focos de parásitos donde los flebotomos se infectasen y lo propagasen por la zona. Cada vez hay más personas con 
tratamientos inmunosupresores que son susceptibles de contraer la enfermedad por lo que la prevención y el tratamiento en los perros, sobre todo en núcleos zoológicos, es fundamental.

El precio es la principal causa de esta sustitución y la legislación no está en sintonía con la situación actual de la enfermedad y prevalencia del parásito en la costa mediterránea, por lo que es necesario cambiar dicha legislación o añadir algún tipo de ayuda para que los núcleos zoológicos y que los propietarios con menos recursos puedan acceder a estos tratamientos a menor coste.

Las autoridades sanitarias han de tener esto en cuenta y valorar si la legislación actual se ajusta a las necesidades reales de la población o si está perjudicando sanitariamente no ya a los animales, sino a los propios humanos.

Los farmacéuticos deberían estar informados de esta problemática y sería recomendable que dispusiesen de las herramientas legales para, en el caso de necesidad, poder recomendar la sustitución de la presentación veterinaria por la humana si no hay posibilidad de dispensar la primera, aunque el motivo sea puramente económico. Tales medidas podrían basarse en autorizaciones de las autoridades competentes en Asuntos Sociales que permitiesen la adquisición de los productos a aquellos consumidores que de verdad lo necesiten, así como la fabricación de formulaciones magistrales para los núcleos zoológicos autorizados por tales medidas hipo- téticas. De esta manera podría protegerse la salud pública.

\section{Conclusión}

Los propietarios particulares están buscando en internet medicamentos para humanos por ser más baratos que los de prescripción veterinaria. Los veterinarios son conscientes de esta problemática y dentro de las competencias que tienen asignadas, tratan de ayudar ofreciendo o mencionando alternativas a los propietarios y a los responsables de los refugios.

\section{Referencias bibliográficas}

1. World Health Organization [Sede web] Pág. de ref. de la OMS, actualizada en 2018, consultada en noviembre de 2018. Leishmaniasis. Disponible en: http://www.who.int/topics/leishmaniasis/en/

2. Carmen Amela, Berta Suarez, Beatriz Isidoro, et al. Evaluación del riesgo de transmisión de L. Infantum en España. Doc. elaborado por el Centro de Coordinación de Alertas y Emergencias sanitarias (CCAES). Ministerio de Sanidad, Servicios Sociales e Igualdad, 23-10-2012. Disponible en: https://www.mscbs.gob.es/profesionales/saludPublica/ccayes/analisisituacion/doc/leishmania.pdf

3. Gaetano 0, Roura X, Crotti A, Maroli $\mathrm{M}$ et al. Guidelines for treatment of leishmaniasis in dogs. JAVMA 2010, 236 (11): 1192-1198. doi:10.2460/javma.236.11.1192

4. Solano-Gallego L, Koutinas A., Miró $\mathrm{G}$, et al. Directions for the diagnosis, clinical staging, treatment and prevention of canine leishmanio- sis. Vet Parasitol 2009, 165: 1-18. doi:10.1016/j.vetpar.2009.05.022

5. Vademécum.com [sede web] Vidal Vademecum Spain I. Cochamb, 24. 28016 Madrid, España. Consultada en noviembre de 2018. www.vademecum.com

6. Werneck, Guilherme L.; Costa, Carlos H. N.; Amorim de Carvalho, Fernando Aecio; et ál. Effectiveness of Insecticide Spraying and Culling of Dogs on the Incidence of Leishmania infantum Infection in Humans: A Cluster Randomized Trial in Teresina, Brazil. PLOS NEGLECTED TROPICAL DISEASES Vol. $8 \quad \mathrm{~N}^{\circ}:$ : 10, Art e3172. OCT 2014. doi:10.1371/journal.pntd. 0003172

7. Pennisi MG. Leishmaniosis of companion animals in Europe: an update. Vet Parasitol. 2015;208:35-47. doi:10.1016/j.vetpar.2014.12.023

8. Lladro, S.; Picado, A.; Ballart, C.; et ál. Management, prevention and treatment of canine leishmaniosis in north-eastern Spain: an online questionnaire-based survey in the province of Girona with special emphasis on new preventive methods (CaniLeish vaccine and domperidone). VETERINARY RECORD Vol. 180 №: 2. JAN 2017. doi:10.1136/vr.103653

9. Comisión de Estándares Biológicos de la OIE. ed. 2004. Manual de las pruebas de diagnóstico y las vacunas para los animales terrestres, Vol. 1. España. Disponible en: http://www.oie. int/doc/ged/d6508.pdf

10. Real Decreto 1132/2010, de 10 de septiembre, por el que se modifica el Real Decreto 109/1995, de 27 de enero, sobre medicamentos veterinarios. Artículo 81. Boletín Oficial del Estado $n^{\circ} 233$ de 25/09/2010. Pag 81447 a 81463 . Disponible en: https://www.boe.es/buscar/doc.php?i$\mathrm{d}=$ B0E-A-2010-14657 